\title{
Microsurgery for Spetzler-Ponce Class A and B arteriovenous malformations utilizing an outcome score adopted from Gamma Knife radiosurgery: a prospective cohort study
}

\author{
Michael K. Morgan, MD, ${ }^{1}$ Markus K. Hermann Wiedmann, MD, ${ }^{1}$ Marcus A. Stoodley, PhD, ${ }^{1}$ and \\ Gillian Z. Heller, $\mathrm{PhD}^{2}$
}

Departments of ${ }^{1}$ Clinical Medicine and ${ }^{2}$ Statistics, Macquarie University, Sydney, New South Wales, Australia

\begin{abstract}
OBJECTIVE The purpose of this study was to adapt and apply the extended definition of favorable outcome established for Gamma Knife radiosurgery (GKRS) to surgery for brain arteriovenous malformations (bAVMs). The aim was to derive both an error around the point estimate and a model incorporating angioarchitectural features in order to facilitate comparison among different treatments.
\end{abstract}

METHODS A prospective microsurgical cohort was analyzed. This cohort included patients undergoing embolization who did not proceed to microsurgery and patients denied surgery because of perceived risk of treatment. Data on bAVM residual and recurrence during long-term follow-up as well as complications of surgery and preoperative embolization were analyzed. Patients with Spetzler-Ponce Class C bAVMs were excluded because of extreme selection bias. First, patients with a favorable outcome were identified for both Class A and Class B lesions. Patients were considered to have a favorable outcome if they were free of bAVM recurrence or residual at last follow-up, with no complication of surgery or preoperative embolization, and a modified Rankin Scale score of more than 1 at 12 months after treatment. Patients who were denied surgery because of perceived risk, but would otherwise have been candidates for surgery, were included as not having a favorable outcome. Second, the authors analyzed favorable outcome from microsurgery by means of regression analysis, using as predictors characteristics previously identified to be associated with complications. Third, they created a prediction model of favorable outcome for microsurgery dependent upon angioarchitectural variables derived from the regression analysis.

RESULTS From a cohort of 675 patients who were either treated or denied surgery because of perceived risk of surgery, 562 had Spetzler-Ponce Class A or B bAVMs and were included in the analysis. Logistic regression for favorable outcome found decreasing maximum diameter (continuous, $\mathrm{OR} 0.62,95 \% \mathrm{Cl} 0.51-0.76$ ), the absence of eloquent location (OR $0.23,95 \% \mathrm{Cl} 0.12-0.43$ ), and the absence of deep venous drainage (OR $0.19,95 \% \mathrm{Cl} 0.10-0.36$ ) to be significant predictors of favorable outcome. These variables are in agreement with previous analyses of microsurgery leading to complications, and the findings support the use of favorable outcome for microsurgery. The model developed for angioarchitectural features predicts a range of favorable outcome at 8 years following microsurgery for Class A bAVMs to be $88 \%-99 \%$. The same model for Class B bAVMs predicts a range of favorable outcome of $62 \%-90 \%$.

CONCLUSIONS Favorable outcome, derived from GKRS, can be successfully used for microsurgical cohort series to assist in treatment recommendations. A favorable outcome can be achieved by microsurgery in at least $90 \%$ of cases at 8 years following microsurgery for patients with bAVMs smaller than $2.5 \mathrm{~cm}$ in maximum diameter and, in the absence of either deep venous drainage or eloquent location, patients with Spetzler-Ponce Class A bAVMs of all diameters. For patients with Class $B$ bAVMs, this rate of favorable outcome can only be approached for lesions with a maximum diameter just above $6 \mathrm{~cm}$ or smaller and without deep venous drainage or eloquent location.

https://thejns.org/doi/abs/10.3171/2016.8.JNS161275

KEY WORDS brain; arteriovenous malformation; vascular disorders; surgery; stereotactic radiosurgery; cohort study

ABBREVIATIONS AVM = arteriovenous malformation; $b A V M=$ brain AVM; CTA = CT angiography; $D S A=$ digital subtraction angiography; GKRS = Gamma Knife radiosurgery; MRA = MR angiography; $\mathrm{mRS}=$ modified Rankin Scale; RCT = randomized controlled trial; VRAS = Virginia Radiosurgery AVM Scale.

SUBMITTED May 17, 2016. ACCEPTED August 3, 2016.

INCLUDE WHEN CITING Published online December 23, 2016; DOI: 10.3171/2016.8.JNS161275. 
$\mathrm{P}$ ATIENTS with brain arteriovenous malformations (bAVMs), and their clinicians, often need to choose from a number of alternative management options, including microsurgery, radiosurgery, embolization, combined intervention, and conservative treatment. However, the risks for each of these options and what is considered success vary. Important considerations in the success of bAVM treatment by microsurgery encompass both the risk of complications and the risk of residual AVM or recurrence. The maximum lesion diameter, location in eloquent brain, deep venous drainage, diffuse nature of the AVM, patient age, history of hemorrhage, and the presence of lenticulostriate feeding artery supply influence the risk of complications of surgery, while young age and the presence of deep venous drainage predict a greater likelihood of recurrence. , $^{2,10,11,14,16,17,24,25}$ Important considerations in the success of bAVM treatment by Gamma Knife radiosurgery (GKRS) encompass the likelihood of predicting obliteration and the likelihood of developing radiation-induced complications as well as the time to obliteration, for which exposure to the natural history of hemorrhage from the bAVM continues. Marginal dose of radiation predicts the obliteration rate; volume of brain exposed to $12 \mathrm{~Gy}$ or more of radiation, location of bAVM (thalamus, basal ganglia, or brainstem), and patient age predict radiation-induced complications; and presentation with hemorrhage or presence of associated proximal intracranial aneurysms predicts a greater likelihood of hemorrhage between treatment and obliteration. ${ }^{5,8,9,20,27}$ Comparing outcomes of treatment for bAVM between microsurgery and GKRS is challenging due to differences in these characteristics between these 2 management pathways and differences in the time course over which problems occur. While randomized control trials (RCTs) would be the best study method for making such comparisons, none exist to compare treatments.

A recent combined cohort series of 2236 patients treated by GKRS validated a grading system, the Virginia Radiosurgery AVM Scale (VRAS), developed from a singlecenter cohort, for estimating the likelihood of favorable outcome. This study defined favorable outcome as bAVM obliteration without postradiosurgery hemorrhage or permanent GKRS-associated symptoms following radiosurgery. ${ }^{27,28}$ Favorable outcome was reported for $81 \%, 75 \%$, $65 \%, 47 \%$, and $41 \%$ of Grades 0 through 4, respectively, at a mean of 7 years following treatment. ${ }^{27}$

Starke and colleagues found that Spetzler-Martin grades predict favorable outcome. ${ }^{28}$ This large GKRS study presents an opportunity to explore whether favorable outcome can be applied to microsurgical cohorts and thus facilitate cautious comparison between microsurgery and GKRS to select the treatment option best suited to the individual patient. A systematic review failed to find any other reported microsurgical series that could incorporate all variables (associated with complications of microsurgery, residual bAVM, or recurrence in long-term follow-up) necessary to establish favorable outcome rates.

From our previous studies we have found that results from surgery for Spetzler-Ponce Class A (Spetzler-Martin Grade I and II) and Spetzler-Ponce Class B (SpetzlerMartin Grade III) bAVMs could be generalized to all our cases of Spetzler-Ponce Class A and Class B bAVMs after incorporating a sensitivity analysis that included nonoperated cases. ${ }^{25}$ However, for Spetzler-Ponce Class C (Spetzler-Martin grade greater than III), there was such bias in selection for treatment, that the results of surgery could not be generalized to apply to all Spetzler-Ponce Class C cases. ${ }^{711}$ For Spetzler-Ponce Class A and B we confirmed that maximum diameter (as a continuous variable), location in eloquent brain, and presence of deep venous drainage predicted the risk of adverse outcomes. ${ }^{11}$ However, our reports do not account for residual and recurrence, an important inclusion in the favorable outcome of GKRS.

We have previously reported our adverse outcomes in a way that allows comparison with other surgical series s.11 $^{2}$ and also by a complication per effectively treated bAVM model that could be used in comparing microsurgery with radiosurgery and embolization but, as yet, has not been adopted for such comparisons. ${ }^{18} \mathrm{We}$, or authors of any other microsurgical cohort series, have not previously reported results in a similar way to that of the VRAS validation study with a method to establish favorable outcome.

The aim of this study was to analyze cases of SpetzlerPonce Class A and B bAVMs treated by microsurgery utilizing a definition of favorable outcome similar to that used by Starke and colleagues in their GKRS series. ${ }^{28} \mathrm{Fa}-$ vorable outcome for our surgical cohort was considered to apply to patients free of bAVM (incorporating cases with an absence of bAVM-related hemorrhage following surgery, residual, or recurrence) and no complications from either embolization or surgery (including those complications from embolization that prevented patients from progressing to intended surgery) that led to a modified Rankin Scale (mRS) score greater than 1 at 12 months following surgery. Because the purpose of this study is intended to assist with management pathway decisions, all patients should be accounted for in the analysis. Therefore, we included patients who were denied surgery because of perceived surgical risk. As a result, we performed a sensitivity analysis to assign such patients to the group of patients not obtaining a favorable outcome. The comparisons were stratified by Spetzler-Ponce class. ${ }^{25}$ The aim of the analysis was to derive both an error around the point estimate and a model incorporating angioarchitectural features. This, then, would facilitate the identification of a group of patients that may do well with treatment by surgery.

\section{Methods}

This study was approved by the institutional human ethics committee and was performed in accordance with institutional ethics committee guidelines.

\section{Data Collection and Study Cohort}

The senior author (M.K.M.) prospectively collected a bAVM database that includes both patients undergoing microsurgery and those not undergoing surgery. This database has been previously described and reported. . $, 3,11,15-18^{-18}$ The responsibility for entry of data into the database was that of the senior author. However, the database (including the outcome ratings) was accessible to residents, fellows, and occupational therapists at the time of assessment and follow-up. The database contained demographic, clinical, 
imaging, and treatment-related information. Patients were included in the database if they were confirmed to have a bAVM on MRI, CT angiography (CTA), or digital subtraction angiography (DSA). The Spetzler-Martin grade was assigned prior to surgery when sufficient imaging detail had been obtained. The database included prospective decisions regarding the planned treatment. For those patients who did not have surgery, the reason why surgery was not performed was reported. The reasons reported for not performing surgery included a decision to be treated elsewhere or by some other means, comorbidities, age greater than 70 years, and poor neurological condition (as a consequence of hemorrhage). The period for which this database captured patients was from 1989 until May 2014 (the last month for surgery by the senior author).

The cohort included patients who underwent microsurgery (including those treated with preoperative embolization), those who underwent embolization but did not proceed to microsurgery due to complications of embolization, and those denied surgery because of perceived risk of surgery who would otherwise be candidates for surgery (i.e., age not greater than 70 years and no significant comorbidities or neurological deficits). Those denied surgery because of perceived risk of surgery were allocated a result as not having had a favorable outcome.

Cases excluded from the analysis involved patients who elected to be treated elsewhere or by some other means despite a recommendation for surgery, those who were partially treated before referral to the senior author, and those for whom the reason for denying surgery was not because of the perceived risk of surgery (i.e., age greater than 70 years, significant comorbidities, or significantly disabled by neurological deficits). These excluded cases have been reported previously. ${ }^{11}$

\section{Favorable Outcomes}

For the microsurgery cohort "favorable outcome" was assigned to patients free of bAVM (both on imaging and absence of bAVM-related hemorrhage following surgery) who had no complications from either preoperative embolization or microsurgery. A complication was defined as a new permanent neurological deficit (as determined within the first 6 weeks of surgery) with an mRS score greater than 1 (assigned at 12 months following surgery) due to preoperative embolization or surgery. Patients who underwent embolization as a prelude to surgery but had complications preventing progress to surgery could be identified and were judged to have not had a favorable outcome. Patients denied surgery because of perceived surgical risk (with age not greater than 70 years and no significant comorbidities or neurological deficits) were included and were judged to not have had a favorable outcome. There are 2 definitions that need further explanation: 1) clinical assessment contributing to favorable outcome and 2) residual or recurrence assessment contributing to favorable outcome.

\section{Clinical Assessment Contributing to Favorable Outcome}

Outcome assessment was performed using the mRS, which was administered preoperatively and at follow-up visits. ${ }^{23,29}$ An adverse outcome was considered a new per- manent neurological deficit assigned within 6 weeks of surgery, and the mRS score was assigned at 12 months (or sooner when the patient had less than 12 months of followup). All patients discharged from the hospital following surgery were followed on at least 1 occasion in clinic by the senior author. All follow-up imaging, from which evidence of recurrence or residual was sought, was reviewed by the senior author as well as the imaging team. The follow-up assessments were usually performed by the senior author, residents, and fellows. Due to the distance of a patient's residence from the clinic, occasionally, the followup review data were supplied to the senior author by the patient's local neurosurgeon or neurologist. For patients who did not undergo surgery, the reason for exclusion was documented prospectively as either perceived greater risk (included in the sensitivity analysis described below) or the patient's having declined recommended treatment, having obtained treatment elsewhere, or having been unsuitable for surgery because of neurological condition or comorbidities (excluded from analysis). These cases have been described previously. ${ }^{11}$

\section{Residual or Recurrence Assessment Contributing to Favorable Outcome}

Residual or recurrence was identified from delayed follow-up radiology specifically performed to detect bAVM (DSA, MR angiography [MRA], or CTA). All patients surviving surgery underwent postoperative DSA within 10 days of surgery. Until 1998, patients underwent 1 postoperative DSA study between 2 and 5 years after surgery. From 1998 onwards, DSA was only performed in the event that MRA raised concern that a bAVM recurrence could not be excluded. Because of the frequency with which MRA scans were performed, the length of imaging follow-up is not significantly different from that of clinical follow-up. ${ }^{18}$ However, to further evaluate the difference between recurrence determined by number of days of clinical follow-up and recurrence determined by number of days of imaging follow-up, Kaplan-Meier curves were compared for these 2 follow-up methods. This was done commencing 30 days following surgery (i.e., beyond the perioperative period but including all clinical follow-up episodes).

\section{Relationship Between Length of Follow-Up and Favorable Outcome}

For the patients included in the regression analysis for favorable outcome, separate Kaplan-Meier curves were generated for those with Spetzler-Ponce Class A and Spetzler-Ponce Class B bAVMSs. The event was considered to be the first detection of not having a favorable outcome and the patients were censored as of the last day of clinical follow-up. Patients denied surgery because of perceived surgical risk were included and considered to have had an event on Day 1.

\section{Analysis of Variables Influencing Favorable Outcome}

Included in the variables chosen for the regression analysis were maximum diameter of the bAVM (as a continuous variable), presence of deep venous drainage, and 
TABLE 1. Summary of demographic and clinical characteristics of patients

\begin{tabular}{|c|c|}
\hline Characteristic & Value \\
\hline Total no. of patients enrolled in database & 778 \\
\hline No. of patients excluded from analysis* & 103 \\
\hline No. of patients included in analysis $†$ & 675 \\
\hline Male sex & $348(52 \%)$ \\
\hline \multicolumn{2}{|l|}{ Age, yrs } \\
\hline Mean \pm SD & $37 \pm 16$ \\
\hline Median (range) & $36(0.8-80)$ \\
\hline Presentation w/ hemorrhage & $307(45 \%)$ \\
\hline Associated proximal intracranial aneurysms & $74(11 \%)$ \\
\hline \multicolumn{2}{|l|}{ Max AVM diameter, $\mathrm{cm}$} \\
\hline Mean \pm SD & $3.4 \pm 1.7$ \\
\hline Median (range) & $3.0(0.3-12)$ \\
\hline \multicolumn{2}{|l|}{ AVM diameter } \\
\hline$<3 \mathrm{~cm}$ & $279(41 \%)$ \\
\hline $3-6 \mathrm{~cm}$ & $350(52 \%)$ \\
\hline$>6 \mathrm{~cm}$ & $46(6.8 \%)$ \\
\hline DVD & $264(39 \%)$ \\
\hline Eloquent AVM location & $297(44 \%)$ \\
\hline \multicolumn{2}{|l|}{ Distribution by Spetzler-Ponce class } \\
\hline A & $359(53 \%)$ \\
\hline $\mathrm{B}$ & $203(30 \%)$ \\
\hline C & $113(17 \%)$ \\
\hline \multicolumn{2}{|l|}{ Follow-up since treatment, mos } \\
\hline Mean \pm SD & $44.4 \pm 46.8$ \\
\hline Median (range) & $27.3(0-242)$ \\
\hline \multicolumn{2}{|l|}{ Cause of failure to achieve favorable outcome } \\
\hline \multicolumn{2}{|l|}{ Spetzler-Ponce Class A } \\
\hline Complication of surgery & $4(1.1 \%)$ \\
\hline Complication of embolization & $1(0.3 \%)$ \\
\hline $\begin{array}{l}\text { Residual at discharge from surgical hospi- } \\
\text { talization }\end{array}$ & $2(0.6 \%)$ \\
\hline Recurrence & $2(0.6 \%)$ \\
\hline Denied surgery because of perceived risk $\ddagger$ & $0(0 \%)$ \\
\hline All causes & $9(2.5 \%)$ \\
\hline \multicolumn{2}{|l|}{ Spetzler-Ponce Class B } \\
\hline Complication of surgery & $39(19.2 \%)$ \\
\hline Complication of embolization & $0(0 \%)$ \\
\hline $\begin{array}{l}\text { Residual at discharge from surgical hospi- } \\
\text { talization }\end{array}$ & $3(1.5 \%)$ \\
\hline Recurrence & $2(9.9 \%)$ \\
\hline Denied surgery because of perceived risk $\ddagger$ & $4(2.0 \%)$ \\
\hline All causes & $48(24 \%)$ \\
\hline
\end{tabular}

DVD $=$ deep venous drainage.

* Patients who were recommended for surgery but who elected to be treated elsewhere or by nonsurgical treatment.

$\dagger$ All patients recommended treatment by surgery or excluded from surgery because of perceived risk.

$\ddagger$ See text for exclusions. eloquent location (as defined by Spetzler and Martin, to include location in primary sensory cortex, motor cortex, language cortex, internal capsule, diencephalon, brainstem, deep cerebellar nuclei, or cerebellar peduncle). ${ }^{24}$ These were included because they had been identified previously by us as predicting the risk of complications for surgery for patients with Spetzler-Ponce Class A and B bAVMs. ${ }^{11}$ In addition, age (as a continuous variable) and presentation with hemorrhage were also included, as they had been identified previously for all Spetzler-Ponce classes to influence the risk of complications of surgery. ${ }^{10}$ Although diffuse bAVM has also been identified as a risk factor for complications of surgery, ${ }_{10}^{10}$ this variable had not been collected prospectively in the database and, therefore, was not included in the analysis.

\section{Probability of Favorable Outcome}

To examine the angioarchitectural influences on favorable outcome, and thus the potential individual variation from the point estimate for the Spetzler-Ponce class grouping, a probability prediction equation for the development of favorable outcome was generated from the multiple logistic regression equation for the combined Class A and B cases. The equation for probability is given as:

Probability of favorable outcome $=e^{a+b x+c y+d z} /(1+$ $\left.e^{a+b x+c y+d z}\right)$, where $a$ is the intercept and $b, c$, and $d$ are the coefficients from the logistic regression equation corresponding to variables with value $x, y$, and $z$.

\section{Statistical Analyses}

Comparisons between groups were performed using a chi-square, Fisher exact test, and Student t-test, as appropriate. From the variables selected for analysis, multiple logistic regression analyses (backward Wald) were performed. A statistical significance level of 5\% was used throughout. The modified Wald method was used to calculate the $95 \%$ confidence interval for a proportion (www. graphpad.com/quickcalcs/) for the outcome data. Statistical analysis was performed using Prism (version 6, GraphPad Software Inc.) and IBM SPSS Statistics (version 22, IBM Corp.) software.

\section{Results}

From a cohort of 778 patients, 103 patients were excluded because they were treated elsewhere or by some other method or had previous partial treatment elsewhere. There were 675 bAVM patients who had not had prior treatment and were treated by microsurgery, treated with embolization with the intention to proceed with microsurgery, or denied surgery because of perceived risk of surgery. The characteristics of these patients are presented in Table 1.

From the 675 patients who were either treated or denied microsurgery because of perceived risk of surgery, there were 562 with Spetzler-Ponce Class A or B bAVMs. These 562 patients included 4 who were denied surgery because of perceived risk, 1 patient treated by embolization who did not progress to surgery because of complications, and 557 who underwent surgery (51 of whom had preoperative embolization) (Fig. 1). Causes for failing to obtain a favorable outcome following microsurgery for Class A bAVMs 


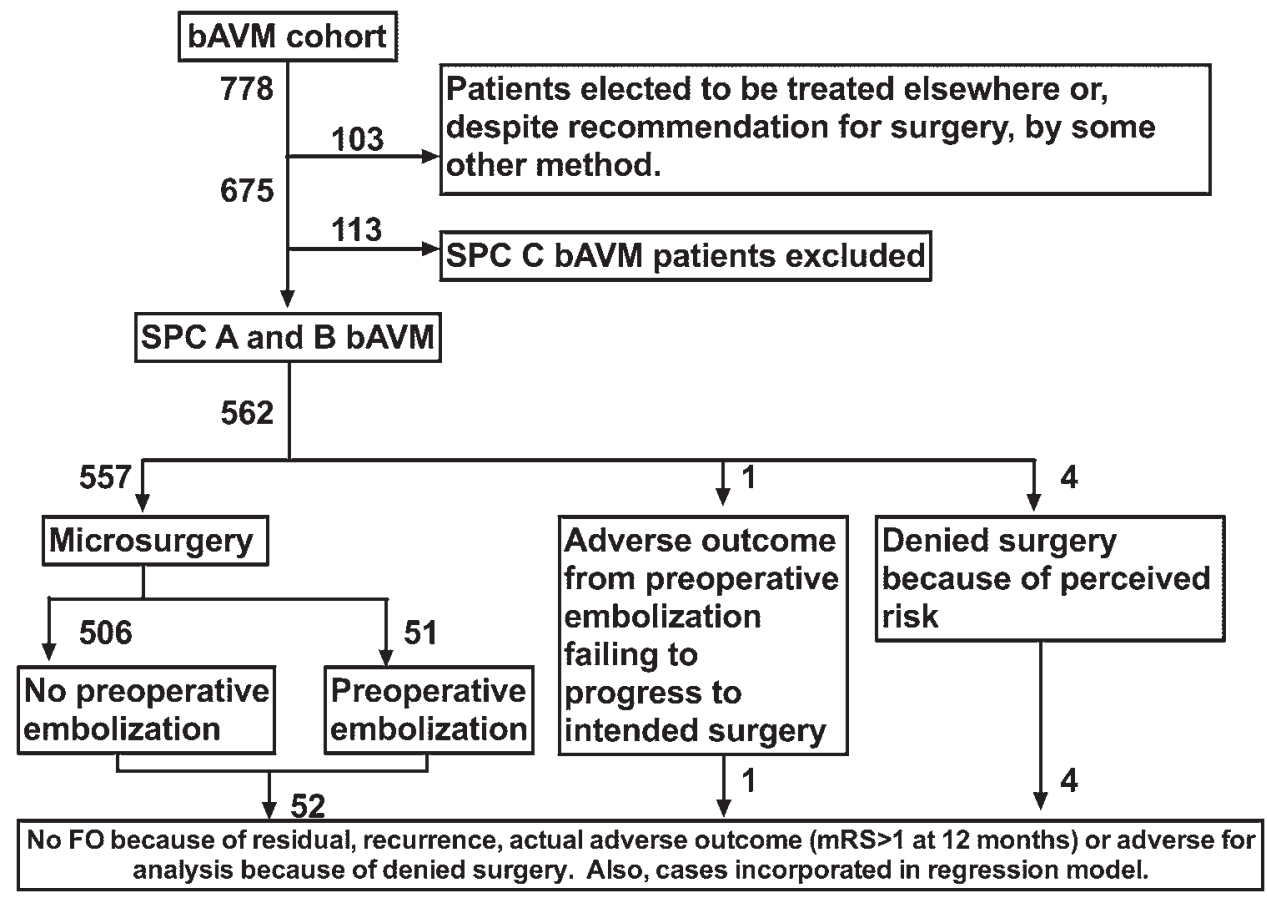

FIG. 1. Inclusion and exclusion criteria and number of patients who did not have a favorable outcome (FO) in the present study for microsurgery of bAVMs. Only patients with Spetzler-Ponce Class (SPC) A and B bAVMs were included. The numbers indicate number of patients. Among the 103 patients excluded as "patients elected to be treated elsewhere or, despite recommendation for surgery, by some other method" were patients that had some partial treatment elsewhere provided by another center and had been referred after failed treatment.

included complications of surgery (1.1\%), complications of embolization $(0.3 \%)$, residual at time of discharge $(0.6 \%)$, and recurrence $(0.6 \%)$. Causes for failing to obtain a favorable outcome following microsurgery for Class B bAVMs included complications of surgery (19\%), residual at time of discharge $(1.5 \%)$, recurrence $(1 \%)$, and denial of surgery because of perceived risk despite the patient's being otherwise a surgical candidate (not older than 70 years, no significant comorbidities, and no major neurological deficit) (2\%) (Table 1 and Fig. 1).

\section{Determination of Favorable Outcome in Microsurgery Cohort}

Recurrence beyond 30 days after microsurgery as detected by clinical follow-up and as detected by radiological follow-up was compared using Kaplan-Meier curves. There was no statistically significant difference between the 2 methods with respect to the detection of recurrence (log-rank Mantel-Cox method, $\mathrm{p}=0.73$ ) (Fig. 2a). Therefore, clinical follow-up was considered reliable in detecting recurrence of bAVM.

For the determination of the appropriate length of follow-up required for favorable outcome for microsurgery, Kaplan-Meier curves of favorable outcome over time for Spetzler-Ponce Class A and B bAVMs are reported in Fig. $2 b)$. Eight-year follow-up data were available for $10 \%$ of the patients initially included in the analysis. Therefore, for estimates of favorable outcome following surgery, the period up to and including 8 years is considered reasonably reliable. For Spetzler-Ponce Class A and B bAVMs, respectively, Kaplan-Meier analysis showed 5-year favor- able outcome rates of $97 \%$ and $77 \%$ and 8 -year favorable outcome rates of $95 \%$ and $77 \%$.

\section{Modeling of Favorable Outcome of Microsurgery for Patients with Class A or Class B bAVMs}

Logistic regression for the combined microsurgery cohort of patients with Spetzler-Ponce Class A or B bAVMs is reported in Table 2. We found that increasing maximum diameter (continuous variable, OR 0.62 , 95\% CI $0.51-$ 0.76 ), location in eloquent brain (OR 0.23 , 95\% CI 0.12 0.43 ), and the presence of deep venous drainage (OR 0.19, 95\% CI 0.10-0.36) were associated with an increased risk of not achieving a favorable outcome. Age and presentation with hemorrhage were not found to influence favorable outcome. This is in accordance with previous analysis, ${ }^{11}$ which used complications arising from microsurgery as the outcome variable, and gives validity to favorable outcome as defined for microsurgery as an outcome measure consistent with previous research.

Probability of favorable outcome from microsurgery for the combined Class A and B bAVMs could be predicted with the following equation, which is graphically represented in Fig. 3: $e^{\text {exponent }} /\left(1+e^{\text {exponent }}\right)$, where exponent $=5.076-(0.473 \times$ maximum diameter $)-1.488$ (if located in eloquent brain) and -1.679 (if deep venous drainage is present).

Because the 3 variables that contribute to the regression analysis are also the 3 variables that constitute SpetzlerMartin grade (and hence the Spetzler-Ponce class), it is possible to identify the predicted favorable outcome within the Spetzler-Ponce class with greater precision because 


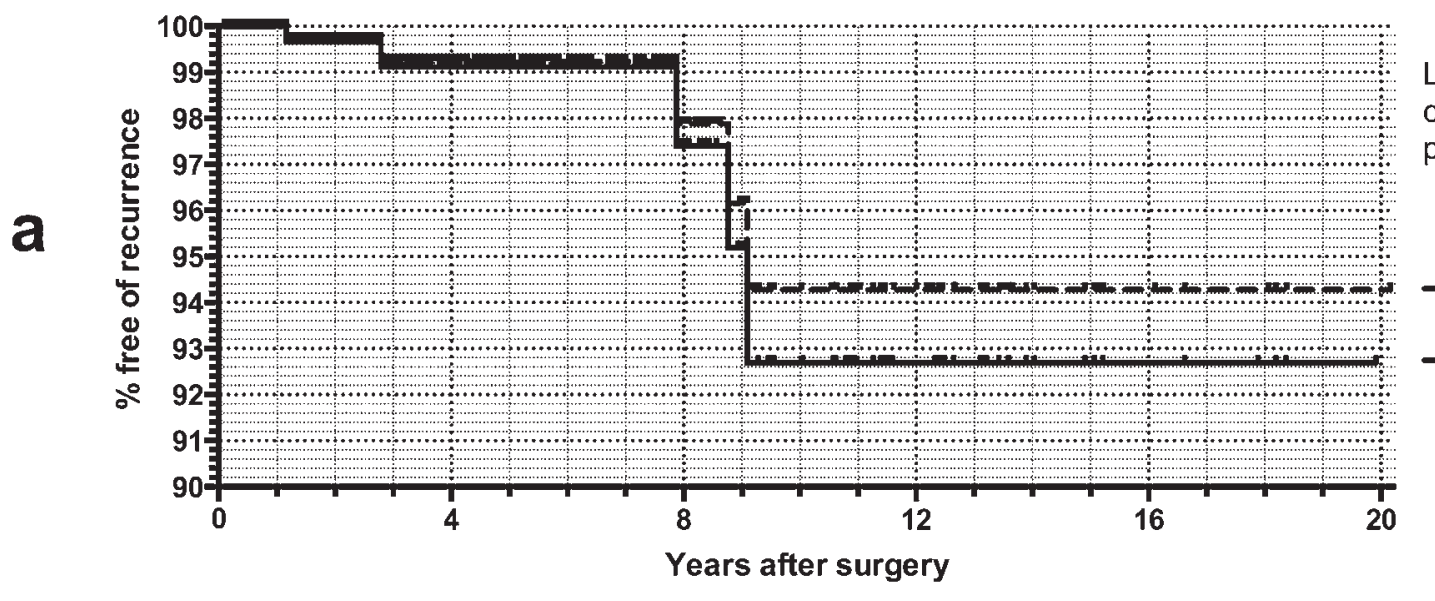

Log-rank (Mantel-Cox) curve comparison: $p=0.73$

- Clinical

Radiological

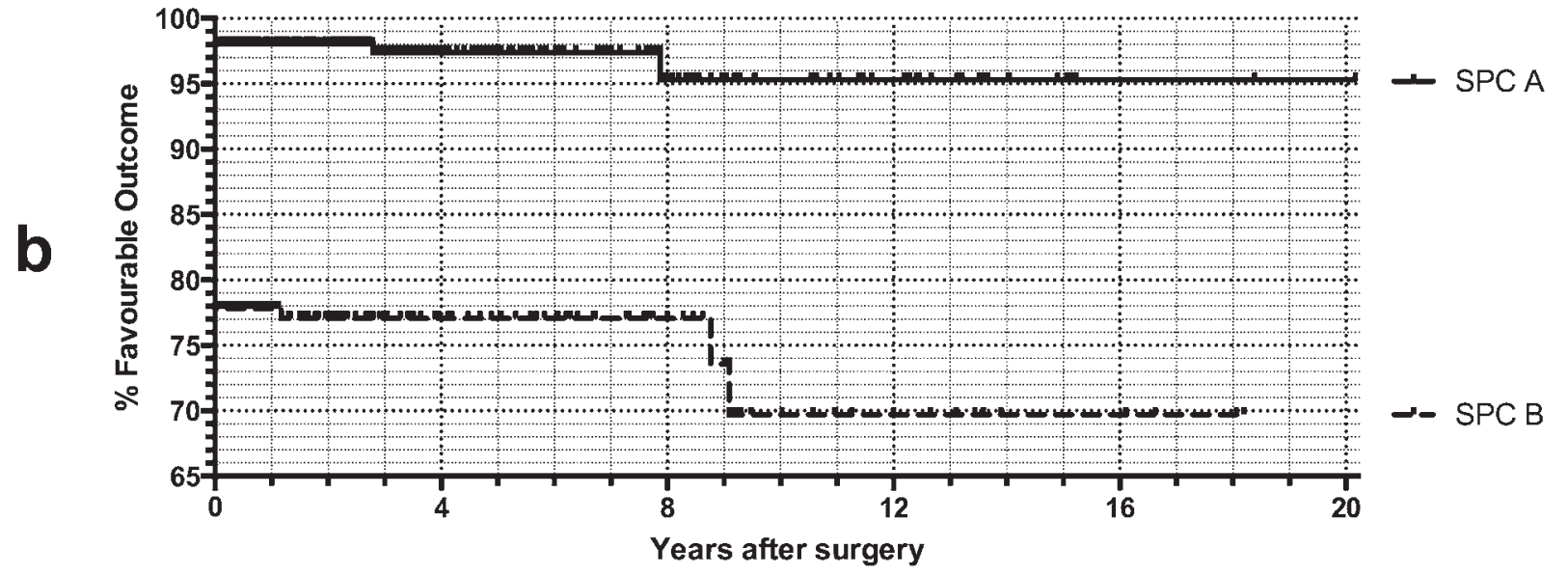

Number at risk comparing method of follow-up (followed 30 days or more)

\begin{tabular}{|c|c|c|c|c|c|c|c|c|c|c|c|c|c|c|c|c|c|c|c|c|c|}
\hline Years & $\mathbf{0}$ & 1 & 2 & 3 & 4 & 5 & 6 & 7 & 8 & 9 & 10 & 11 & 12 & 13 & 14 & 15 & 16 & 17 & 18 & 19 & 20 \\
\hline Clinical follow-up & 493 & 382 & 282 & 198 & 158 & 129 & 106 & 88 & 69 & 54 & 43 & 35 & 29 & 21 & 12 & 9 & 6 & 4 & 3 & 1 & 1 \\
\hline $\begin{array}{r}\text { Radiological } \\
\text { follow-up }\end{array}$ & 470 & 374 & 242 & 168 & 136 & 112 & 92 & 73 & 54 & 41 & 33 & 25 & 20 & 16 & 9 & 7 & 5 & 4 & 3 & 1 & 0 \\
\hline \multicolumn{22}{|c|}{ Number at risk comparing $\mathrm{SPC} A$ with $\mathrm{SPC} B$} \\
\hline Years & $\mathbf{0}$ & 1 & 2 & 3 & 4 & 5 & 6 & 7 & & 9 & 10 & 11 & 12 & 13 & 14 & 15 & 16 & 17 & 18 & 19 & 20 \\
\hline SPCA & \begin{tabular}{|l|}
359 \\
\end{tabular} & 267 & 196 & 133 & 106 & 82 & 65 & 56 & 43 & 33 & 28 & 24 & 19 & 13 & 8 & 5 & 2 & 2 & 2 & 1 & 1 \\
\hline SPC B & 203 & 115 & 87 & 61 & 52 & 47 & 41 & 32 & 26 & 21 & 15 & 11 & 10 & 8 & 4 & 4 & 4 & 2 & 1 & 0 & 0 \\
\hline
\end{tabular}

Percentage Favorable Outcome

\begin{tabular}{|l|c|c|c|c|c|c|c|c|c|c|c|}
\hline SPC A & $\%$ & 98.1 & 98.1 & 97.4 & 97.4 & 97.4 & 97.4 & 97.4 & 95.3 & 95.3 & 95.3 \\
\hline SPC B & $\%$ & 77.8 & 77.1 & 77.1 & 77.1 & 77.1 & 77.1 & 77.1 & 77.1 & 73.6 & 69.7 \\
\hline
\end{tabular}

Comparing SPC A with SPC B Kaplan-Meier curves.

log-rank (Mantel-Cox) $\quad \mathrm{p}<0.001$

Hazard ratio (logrank) $9.5(95 \% \mathrm{Cl}: 5.7-16.9)$

FIG. 2. Kaplan-Meier analyses. The upper graph (a) shows a comparison between methods of reporting follow-up for patients followed for more than 30 days by date of radiological procedure for censor or event (detection of bAVM recurrence) versus clinical follow-up date for censor or event (detection of bAVM recurrence). There is no difference in method of following patients in determining percentage free from recurrence (log-rank, $p=0.73$ ). The lower graph $(b)$ compares the cumulative freedom of favorable outcomes for Spetzler-Ponce Class A and Class B bAVMs. The proportion of patients at risk is more than $10 \%$ for the first 8 years after surgery. At that time point, favorable outcome was present for $95 \%$ of Class A and $77 \%$ of Class B bAVMs. The difference was statistically significant (log-rank $p<0.001, \mathrm{HR} 9.5,95 \% \mathrm{Cl} 5.7-16.9)$.

maximum diameter can be used, rather than utilizing 1 , 2 , or 3 points for the maximum diameters less than $3 \mathrm{~cm}$, $3 \mathrm{~cm}$ to $6 \mathrm{~cm}$, and greater than $6 \mathrm{~cm}$, respectively. Our model predicts a range of favorable outcome for SpetzlerPonce Class A bAVMs between $88 \%$ (for a bAVM with deep venous drainage approaching $3 \mathrm{~cm}$ in maximum diameter) and 99\% (for the smallest bAVM that has neither deep venous drainage nor eloquent location). The same model for Spetzler-Ponce Class B bAVMs predicts a range of favorable outcome between $62 \%$ (for a bAVM that has both deep venous drainage and eloquent location and is approaching $3 \mathrm{~cm}$ in maximum diameter) and $90 \%$ (for a bAVM that has neither deep venous drainage nor eloquent location and is just above $6 \mathrm{~cm}$ in maximum diameter). For the detailed predictions of various combinations of maximum diameter, deep venous drainage, and eloquence, and 
TABLE 2. Results of logistic regression for favorable outcome in microsurgery cohort

\begin{tabular}{|c|c|c|c|c|}
\hline $\begin{array}{c}\text { Characteristic } \\
\text { Analyzed }\end{array}$ & $\begin{array}{c}\mathrm{p} \text { Value from } \\
\text { Univariate Analysis }\end{array}$ & $\begin{array}{c}\beta \text { from Final } \\
\text { Regression Equation }\end{array}$ & $\begin{array}{l}\text { p Value from Multiple } \\
\text { Logistic Regression }\end{array}$ & $\begin{array}{c}\text { OR } \\
(95 \% \mathrm{Cl})\end{array}$ \\
\hline \multicolumn{5}{|l|}{ Patient characteristics } \\
\hline Presentation w/ hemorrhage & 0.25 & & & \\
\hline Age & 0.97 & & & \\
\hline \multicolumn{5}{|l|}{ Brain AVM characteristics } \\
\hline Max diameter ${ }^{*}$ & $<0.001$ & -0.473 & $<0.001$ & $0.62(0.51-0.76)$ \\
\hline Eloquent location & $<0.001$ & -1.488 & $<0.001$ & $0.23(0.12-0.43)$ \\
\hline DVD & $<0.001$ & -1.679 & $<0.001$ & $0.19(0.10-0.36)$ \\
\hline
\end{tabular}

how these compare with the point estimate and $95 \%$ confidence interval of Spetzler-Ponce Class A and B favorable outcome, refer to Fig. 3.

\section{Discussion}

A definition of outcomes, "favorable outcome," incorporating obliteration of bAVM, posttreatment bAVM hemorrhage, and permanent adverse outcomes of treatment has recently been applied to a large series of bAVMs treated with GKRS. We have applied this outcome assessment to microsurgery. ${ }^{27,28}$ We found that microsurgery was associated with favorable outcome rates of 97\% (95\% CI 95\%$99 \%$ ) and $76 \%$ (95\% CI 70\%-82\%) for Spetzler-Ponce Class A and Class B bAVMs, respectively. It is important to not only compare the point estimates of the Spetzler-Ponce class but also consider how an individual's angioarchitecture contributes to influence favorable outcome within a Spetzler-Ponce class in order to come to a decision with regard to recommended treatment. When Spetzler-Ponce Class A and Class B bAVMs treated by microsurgery were combined, increasing maximum diameter (OR 0.62, 95\% CI 0.51-0.76), location in eloquent brain (OR $0.23,95 \%$ CI $0.12-0.43$ ), and the presence of deep venous drainage (OR $0.19,95 \%$ CI $0.10-0.36$ ) were each associated with an increased risk of not achieving a favorable outcome. Age and presentation with hemorrhage were not found to influence the likelihood of favorable outcome. The variability in favorable outcome associated with angioarchitectural features is illustrated in Fig. 3, with favorable outcome rates for Spetzler-Ponce Class A bAVMs ranging between $88 \%$ (for a bAVM with deep venous drainage approaching $3 \mathrm{~cm}$

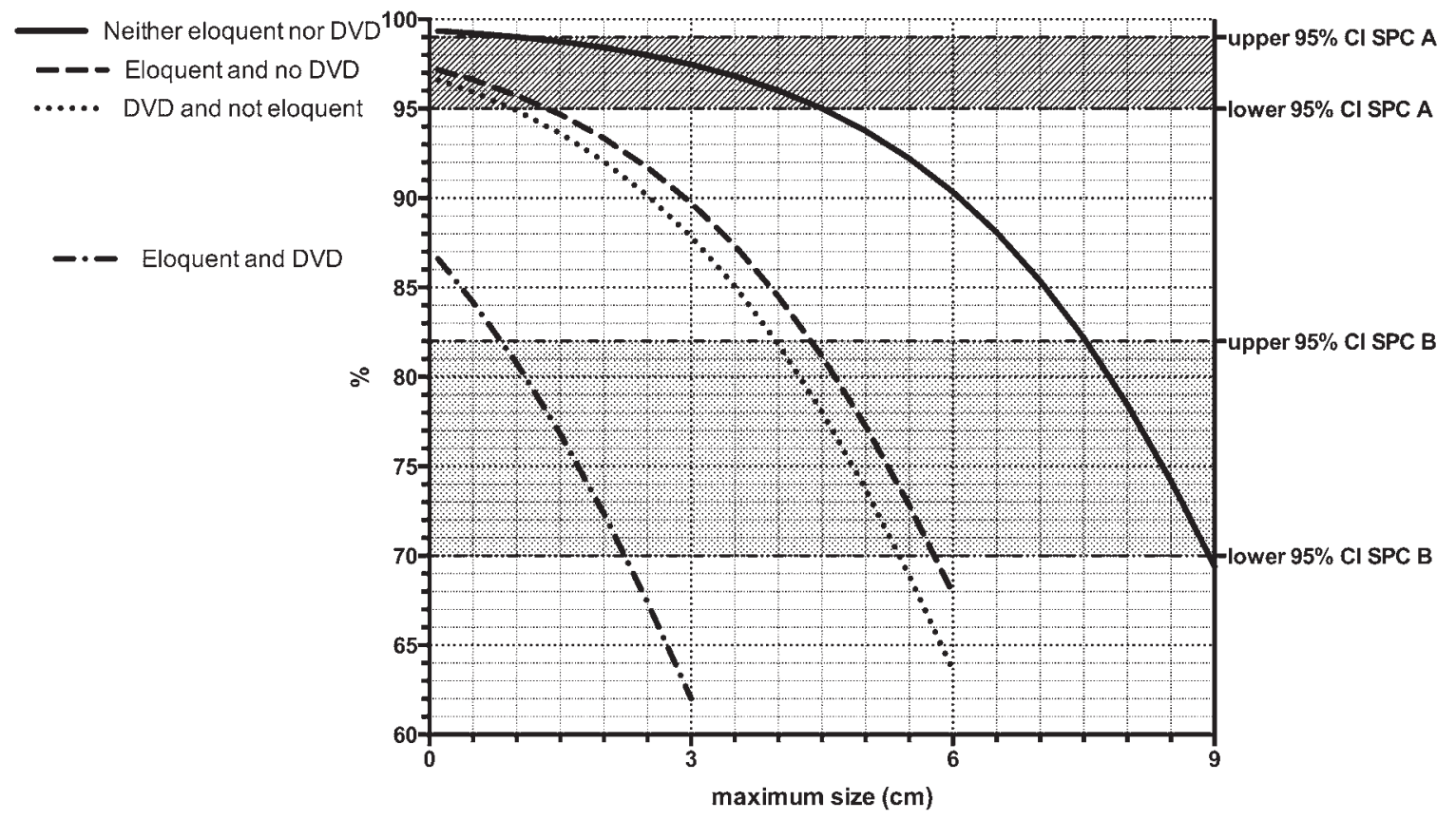

FIG. 3. Regression model of angioarchitectural features (and their combination) in predicting favorable outcome for patients with Spetzler-Ponce Class A or B bAVMs. The y-axis indicates favorable outcome rate (\%). The $95 \%$ confidence intervals of the point estimate of favorable outcome for Spetzler-Ponce Classes A and B are provided to appreciate how the various combinations of maximum diameter, eloquent location, and deep venous drainage (DVD) compare for microsurgery. The outcome lines are discontinued at the boundary with Spetzler-Ponce Class $\mathrm{C}$ because these cases were not incorporated in the regression analysis. Lower $95 \% \mathrm{Cl}=$ lower bound, $95 \% \mathrm{Cl}$; upper $95 \% \mathrm{Cl}=$ upper bound, $95 \% \mathrm{Cl}$. 
in maximum diameter) and $99 \%$ (for the smallest bAVM that has neither deep venous drainage nor eloquent location). For Spetzler-Ponce Class B, the corresponding range of favorable outcome rates is between 62\% (for a bAVM with both deep venous drainage and eloquent location and approaching $3 \mathrm{~cm}$ in maximum diameter) and $90 \%$ (for a bAVM that has neither deep venous drainage nor eloquent location and is just greater than $6 \mathrm{~cm}$ in maximum diameter). From our regression analysis, we predict for our cohort that a $90 \%$ rate of favorable outcome can be achieved for all Spetzler-Ponce Class A bAVMs that are smaller than $2.5 \mathrm{~cm}$ in maximum diameter and that this rate can be achieved for Class A bAVMs of all diameters in the absence of either deep venous drainage or eloquent location.

Because microsurgery and GKRS cohorts vary significantly, presumably on the basis of referral pattern, a comparison with GKRS series can only be made with caution. Therefore, for the report by Starke and colleagues (in which favorable outcomes were reported for GKRS to be $70 \%$ and $61 \%$ for Spetzler-Ponce Class A and B bAVMs, respectively), ${ }^{28}$ from which our definition of outcome was derived, comparison with microsurgery needs to take into consideration differences in cohorts and the weighting of complications early in the microsurgery series. Taking this into consideration, it is reasonable to suggest that patients with Spetzler-Ponce Class A bAVMs who are likely to have at least a $90 \%$ rate of favorable outcomes (i.e., those with Class A bAVMs smaller than $2.5 \mathrm{~cm}$ in maximum diameter and, in the absence of either deep venous drainage or eloquent location, all diameters) are well served by treatment with microsurgery. Similar outcomes may be achieved with GKRS in patients with small, unruptured deeply placed bAVMs. For such patients, particularly those with Spetzler-Ponce Class B bAVMs, GKRS may be the preferred option. However, it is important when making this decision that audited outcomes from centers in which patients are treated by microsurgery reproduce the outcomes reported in our microsurgery series or the GKRS series from which favorable outcome is derived.

\section{Past and Current Practice From Which These Results are Derived}

The practice from which our microsurgical results are derived is heavily biased toward surgery. This is evident in the vast majority of patients with Spetzler-Ponce Class A and $\mathrm{B}$ bAVMs being recommended treatment by microsurgery. The reason for this relates to the senior author's experience with surgery as compared with the available experience in LINAC radiosurgery. GKRS was not available in Australia for the treatment of bAVMs before 2011. However, our series, biased toward treatment by surgery, also offers an opportunity to provide insight into what the results from all similar bAVMs treated by surgery would be with the sensitivity analysis adjustment provided. This is only possible where almost all patients are treated by the one technique. This pattern does not reflect the senior author's current recommendations for treatment, as the evidence would suggest an important role for selected cases to be treated by GKRS. The senior author currently recommends GKRS be considered first for patients with unruptured Spetzler-Ponce Class B bAVMs and for those with a Lawton-Young Scale score greater than 6, providing they are likely to attain a favorable outcome with GKRS. Microsurgery is recommended for the majority of patients with Spetzler-Ponce Class A bAVMs unless they have a Lawton-Young Scale score greater than 6 (see below). With this recent analysis, the senior author recommends that microsurgery be considered first for patients with SpetzlerPonce Class A bAVMs smaller than $2.5 \mathrm{~cm}$ in maximum diameter and, in the absence of either deep venous drainage or eloquent location, Spetzler-Ponce Class A bAVMs of all diameters. Although Class A bAVMs smaller than $2.5 \mathrm{~cm}$ respond better to GKRS than larger lesions, ${ }^{9}$ surgery is also safest and more effective for these smaller bAVMs. We now recommend GKRS for patients with Spetzler-Ponce Class A bAVMs that are $2.5 \mathrm{~cm}$ or greater with either deep venous drainage or eloquent location.

\section{Evidence Underpinning Recommendations of Microsurgery Versus GKRS Treatment for bAVMs}

There are a number of considerations when choosing between microsurgery and GKRS for treating a bAVM. Included in these considerations are complications of treatment, obliteration rate, length of time to obliteration, continued exposure to natural history, and expertise that is available to the patient (local practice). When dealing with so many variables, an approach to assist management comparisons of the highest quality evidence is to match patients and examine outcomes of randomized control trials (RCTs). However, the challenge of an RCT in bAVM management is significant, given that it is a disease state with a detection rate of less than 2 per 100,000 per year, ${ }^{1,6,19,26}$ the reluctance of participants and their clinicians to randomize treatment in an immediately high stakes consequence with irreversible outcome, ${ }^{2,12}$ and the large number of characteristics that predict good outcomes (some of which are unique to each of the treatment methods). As an example, a recent RCT comparing treatment (by any means) with conservative management for unruptured bAVMs, ARUBA (A Randomized Trial of Unruptured Brain Arteriovenous Malformations) was insufficiently powered to compare surgery with GKRS. ARUBA was initially intended to enroll 800 patients but eventually enrolled 226 over 6 years in 39 centers..$^{14}$ Thus, on average no more than 1 patient per year was enrolled from each of these treatment centers considered to have expertise in the management of bAVMs. This highlights the challenges of RCTs in comparing treatment methods for bAVMs.

In the absence of guidelines from RCTs, cohort studies currently provide the best evidence for informing recommendations for treatment. ${ }^{2,12}$ In order for cohort studies to be comparable, it is necessary to understand how selection bias influences management decisions and to have sufficient information available to understand all the necessary outcome considerations (e.g., immediate and delayed complications of treatment, occlusion rates, the natural history until occlusion is achieved, as well as recurrence rates). For bAVMs, there are few methods of directly comparing one management pathway from cohort studies with another that synthesizes these demands on information. The first of such scores was that of Pollock and Flickinger, who introduced an outcome score that combined both bAVM 
obliteration and absence of neurological deficit ("excellent").20,21 Recently, Pollock and colleagues reported that an excellent result was achieved in $71 \%$ (95\% CI 67\%$76 \%$ ) of 381 patients treated by GKRS, of whom $42 \%$ and $41 \%$ had Spetzler-Ponce Class A and Class B bAVMs, respectively. ${ }^{22}$ Nevertheless, these results were not analyzed in terms of excellent outcomes by Spetzler-Ponce class. Starke and colleagues have analyzed a large series of patients with Spetzler-Ponce Class A and B bAVMs treated by GKRS utilizing outcomes as defined by favorable outcome. ${ }^{28}$ Overall, they reported $66 \%$ favorable outcome (95\% CI 63\%-69\%) in 928 patients with Spetzler-Ponce Class A and B bAVMs. ${ }^{28}$ Although these results appear slightly worse than those reported by Pollock and colleagues, ${ }^{22}$ when incorporated within data analyzed from 8 institutions and obtained from cases involving 2236 patients (of whom the University of Virginia contributed 1012 patients), the overall favorable outcome rate was $60 \%$, suggesting that the results from the cases contributed to the study by the University of Virginia (the only participating site in which results of favorable outcome could be analyzed by Spetzler-Ponce class) can reasonably be generalized to other major GKRS centers. ${ }^{27}$ Of importance in the assistance to treatment decision making, a cohort series by Starke and colleagues (the VRAS validation study), involving a large number of patients treated with GKRS, used an outcome measure of favorable outcome that can also be applied to surgical cohort series. ${ }^{27}$

Similar to the difficulties of GKRS outcome scores being applied to surgical series, surgical series have difficulty in translating outcomes that can be applied to GKRS series. This is because of the concentration on complications of surgery alone without taking into consideration obliteration rates measured over time. The Spetzler-Ponce class has been demonstrated to be an excellent predictor for adverse outcome and has been previously validated as generalizable to all similarly graded bAVMs (whether surgically treated or not) for patients eligible for surgery (i.e., not older than 70 years and without major comorbidities or severe neurological deficits).,11 The weighting of the components of the variables also reflects the relative weighting in a regression equation. ${ }^{11}$ The Spetzler-Ponce class, condensing the Spetzler-Martin grade into Class A (SpetzlerMartin Grades I and II), Class B (Spetzler-Martin Grade III), and Class C (Spetzler-Martin grade greater than III), has strong predictive values for complications and simplifies the decision-making process into generally suitable for surgery (Spetzler-Ponce Class A) and generally unsuitable for surgery (Spetzler-Ponce Class C) ${ }^{23}$ For patients with Spetzler-Ponce Class B bAVMs, the decision as to which treatment modality is unclear is further assisted by applying the Lawton-Young scale, which incorporates age (1, 2 , or 3 points for ages less than 20 years, 20 to 40 years, and more than 40 years, respectively), presentation without hemorrhage (an additional point), and AVM diffuseness (an additional point) into stratifying Spetzler-Ponce Class B bAVMs into high and low risk for microsurgery (high risk would be indicated by a combined score of more than 6 points, obtained by summing the Spetzler-Martin grade and the Lawton-Young scale score).$^{10,13}$ However, grading scales fail to account for residual and recurrent bAVMs over time and therefore are not designed to compare the likely outcome expected from microsurgery with that expected from GKRS. The difficulty in making such comparisons is underscored by differences in obliteration rates, time to obliteration, complication rates, and time to complication development. However, adapting the definition of favorable outcome used in the GKRS series reported by Starke and colleagues ${ }^{26}$ (bAVM obliteration without postradiosurgery hemorrhage or permanent GKRS-associated symptoms) to a surgical cohort incorporating obliteration rates over time (bAVM obliteration without recurrence or permanent neurological deficit from treatment) facilitates cautious comparison. Our study is the only microsurgical series that incorporates cases in which surgery was not performed because of perceived risk and also includes the presence of residual or recurrence over the follow-up period.

Our results suggest that microsurgery is generally superior to GKRS with respect to achieving favorable outcome. However, variability in how angioarchitectural features influence outcome within a Spetzler-Ponce class is also a major consideration in assisting individualized decision making. Of course, there are a number of limitations to our study and conclusions that need to be considered.

\section{Limitations}

The limitations of our cohort, including referral selection bias, have been discussed previously. ${ }^{2,3,11,18}$ The most important of these limitations is that our results are from a retrospective analysis of a prospectively designed and maintained database, rather than an RCT. However, as discussed above, there are no RCT results available, and the difficulties of performing an RCT make a good-quality $\mathrm{RCT}$ unlikely in the near future. In addition to the quality of evidence, there are specific limitations that need to be discussed if comparisons are to be made between our results and those from GKRS series.

\section{Angioarchitectural Differences Between Microsurgery and GKRS Cohorts}

There are angioarchitectural differences between surgical series and GKRS series. As an example, the cohort of cases from Starke and colleagues' GKRS study has a greater proportion of smaller, deeply placed bAVMs. ${ }^{28}$ This selection bias is to be expected. However, bAVMs can be categorized by common variables (including size and deep venous drainage) predicting outcomes, making comparison possible despite the overall differences in the 2 cohorts. The importance of being able to stratify patients by Spetzler-Ponce class decreases the impact of this problem in comparing these cohorts, and the predictive model (Fig. 3 ) allows an understanding of how variations in angioarchitectural features may cause an individual to be more, or less, likely to attain a favorable outcome as compared with the point estimate $95 \%$ confidence interval range within the Spetzler-Ponce class.

\section{Length of Follow-Up}

The lengths of follow-up differ between the GKRS series and our microsurgery series. The mean duration of follow-up in our microsurgery series was 44 months, which is considerably shorter than the corresponding duration of 7 years in the GKRS series. However, the GKRS 
series of Starke and colleagues excluded cases in the first 2 years (except for those sustaining adverse outcomes) and this was not done for the microsurgery series. ${ }^{28}$ Exclusion of cases during the first 2 years would be inappropriate for microsurgical series but is appropriate for GKRS series given that obliteration does not occur immediately following GKRS. By examining the implication of time on favorable outcome following microsurgery in our Kaplan-Meier curve analysis, we could reasonably report the proportion with favorable outcome as being reliable for the first 8 years following microsurgery (i.e., a length of follow-up comparable to that of the GKRS series, which had a mean of 7 years) with more than $10 \%$ of the initial cohort remaining. Over this period, the favorable outcome rate for microsurgery of Spetzler-Ponce Class A bAVMs declined to $95 \%$ and that for Spetzler-Ponce Class B bAVMs to $77 \%$. This differs only slightly from the results as calculated from the overall rates of favorable outcome in our cohort of $97 \%$ (95\% CI 95\%-99\%) and 76\% (95\% CI 70\%-82\%) for microsurgical treatment of Class A and Class B bAVMs, respectively. Therefore, favorable outcome results were sustainable from microsurgery and our conclusions with regard to microsurgery are likely to hold for of the first 8 years following resection. From our previous work, it would be reasonable to expect little change to the recurrence rate following surgery for bAVM with time for either adults or with children without deep venous drainage. ${ }^{17}$ However, children with deep venous drainage had a significant rate of recurrence and these patients may require a more prolonged follow-up to discern the longterm favorable outcome proportion. ${ }^{17}$

\section{Definition of Favorable Outcome}

Another limitation in facilitating a comparison between treatment by microsurgery and treatment by GKRS is that the definition of favorable outcome used in our study is similar but not equivalent to the definition employed by Starke and colleagues in their GKRS series. ${ }^{28}$ For our study, the definition of favorable outcome was no residual or recurrent bAVM and no complications from either embolization or surgery (including those complications from embolization that prevented the patient from progressing to intended surgery) leading to a new permanent neurological deficit determined within 6 weeks of surgery with an mRS score greater than 1 at 12 months after surgery (or at last follow-up in cases in which 12 months' followup is not achieved). For the GKRS series ${ }^{28}$ the definition of favorable outcome was defined as AVM obliteration without postradiosurgery hemorrhage or permanent GKRS-associated symptoms following radiosurgery. ${ }^{27,28}$ Although these definitions are not equivalent, we believe they are similar. Following microsurgery, there are invariably symptoms of surgery that remain permanent, such as paresthesia around the scar. Therefore, an mRS score of 1 is almost always the minimum score following microsurgery. We believe patients are more focused upon deficits leading to an mRS score greater than 1 and that, hence, our definition is reasonable.

\section{Detection of Residual and Recurrence}

A further limitation in our study is our reliance upon clinical follow-up rather than imaging confirmation of the bAVM absence. However, we did compare those followed by date of imaging investigation with those followed by date of clinical follow-up for cumulative event detection rate (recurrence of bAVM) by Kaplan-Meier curves and verified that there was no statistically significant difference between these 2 methods of analysis in our cohort. Therefore, we believe that utilizing the date of clinical follow-up was reasonable.

\section{Patient Inclusion and Exclusion}

Another unavoidable difference between our results and those of GKRS series, such as the series reported by Starke and colleagues, ${ }^{27,28}$ is the inclusion of patients undergoing previous treatment in the GKRS series. One can reasonably hypothesize that the difference in the outcomes from ARUBA and previously published cohort studies in the management of unruptured bAVMs is due to the morbidity and mortality that arise from the first treatment undertaken that precludes progression to a second treatment. In a retrospectively analyzed cohort, these patients cannot usually be identified and, as a consequence, are not included in the analysis. As an example, a significant percentage of the patients included in the VRAS validation study had previous treatment (embolization, radiotherapy, or surgery in $22 \%, 9 \%$, and $5 \%$ of cases, respectively). ${ }^{27}$ The authors did not report cases in which GKRS had been planned after initial treatment but was not performed..$^{27}$ Therefore, the GKRS validation study may overstate favorable outcomes for cases intended for treatment by GKRS from the time of first diagnosis. On the other hand, our cohort does not include patients previously treated elsewhere and does include patients in whom preoperative embolization was performed but surgery was not performed because of complications arising from embolization. Therefore, we believe that our microsurgery series reflects outcome for these cases planned to be treated by microsurgery identified from the time of first diagnosis.

\section{Sensitivity Analysis}

A further difference between our results and those of the GKRS study is that patients excluded for treatment by GKRS are not included in a sensitivity analysis. This is an unavoidable consequence of the general referral pattern and again needs to be considered when comparing the 2 cohort studies. Our microsurgery cohort was analyzed to include patients who would have been eligible for surgery (i.e., age not greater than 70 years and no major comorbidities or severe neurological deficits) but were denied surgery on the basis of perceived risk. These patients were considered to have not had a favorable outcome in our analysis. Thus, our series incorporates a sensitivity analysis of microsurgery cases. The cases excluded, and reasons for exclusion, have been previously reported. ${ }^{11,18}$ No patients with Spetzler-Ponce Class A bAVM were denied surgery because of perceived surgical risk, 6 of 205 patients with Class B bAVMs were denied surgery because of perceived surgical risk, and 33 of 112 Class C bAVMs were denied surgery because of perceived surgical risk..$^{11,18}$ The large number of exclusions for Class C bAVM emphasizes the significant selection bias exercised in the management of 
these cases and highlights the limitation of useful information that can be derived from including patients with Spetzler-Ponce Class C lesions in any analysis of microsurgery. Similarly, such patients are represented in less than $10 \%$ of VRAS validation study cases and not present at all in the University of Virginia GKRS series. ${ }^{27,28}$ Therefore, comparison of management of Spetzler-Ponce Class $\mathrm{C}$ cases should be made with caution.

\section{Alternative Outcome Measures}

Alternative outcome measures are important, and favorable outcome is only one such tool to be considered in deciding between management pathways. It is important to look at outcomes from a variety of perspectives that facilitate comparison between treatments. Outcome scores should incorporate, wherever possible, measures that take into account differences in feasibility of treatment (i.e., analysis to incorporate those denied treatment by a modality of treatment because of perceived risk), obliteration rates, time to obliteration (and exposure to the continuing risk of hemorrhage), and complications. An example of such a method of comparing microsurgery with GKRS is adopting the accountancy tool of cost-effectiveness, in which the cost or complications (incorporating patients denied surgery because of perceived surgical risk) is reported per effectively treated bAVM. In such an analysis, we have previously reported that at 9 years after surgery there is a $1.4 \%, 24 \%$, and $63 \%$ complication rate per effectively treated bAVM for Spetzler-Ponce Class A, B, and $\mathrm{C}$, respectively. ${ }^{18}$ It will be interesting to see this method of analysis for other treatment modalities.

\section{Conclusions}

By applying a measure of outcome recently validated in a large cohort series for GKRS to that of microsurgery, in which favorable outcome could generally be defined as freedom of bAVM and freedom from adverse outcomes of treatment, microsurgery appears to offer an advantage in general over GKRS. Modeling the impact of angioarchitectural features on favorable outcome can assist in refining the recommendations between treatments by GKRS and microsurgery for the individual patient. For Spetzler-Ponce Class A bAVM, a favorable outcome can be achieved by microsurgery in at least $90 \%$ of bAVMs smaller than $2.5 \mathrm{~cm}$ in maximal diameter and, in the absence of either deep venous drainage or eloquent location, Class A bAVMs of all diameters.

\section{Acknowledgments}

We thank Dr. Elizabeth Anne Ritson, MBBS, for editorial assistance.

\section{References}

1. Al-Shahi R, Bhattacharya JJ, Currie DG, Papanastassiou V, Ritchie V, Roberts RC, et al: Prospective, population-based detection of intracranial vascular malformations in adults: the Scottish Intracranial Vascular Malformation Study (SIVMS). Stroke 34:1163-1169, 2003

2. Bervini D, Morgan MK, Ritson EA, Heller G: Surgery for unruptured arteriovenous malformations of the brain is better than conservative management for selected cases: a prospective cohort study. J Neurosurg 121:878-890, 2014
3. Davidson AS, Morgan MK: How safe is arteriovenous malformation surgery? A prospective, observational study of surgery as first-line treatment for brain arteriovenous malformations. Neurosurgery 66:498-505, 2010

4. Du R, Keyoung HM, Dowd CF, Young WL, Lawton MT: The effects of diffuseness and deep perforating artery supply on outcomes after microsurgical resection of brain arteriovenous malformations. Neurosurgery 60:638-648, 2007

5. Flickinger JC, Kondziolka D, Maitz AH, Lunsford LD: An analysis of the dose-response for arteriovenous malformation radiosurgery and other factors affecting obliteration. Radiother Oncol 63:347-354, 2002

6. Gabriel RA, Kim H, Sidney S, McCulloch CE, Singh V, Johnston SC, et al: Ten-year detection rate of brain arteriovenous malformations in a large, multiethnic, defined population. Stroke 41:21-26, 2010

7. Han PP, Ponce FA, Spetzler RF: Intention-to-treat analysis of Spetzler-Martin grades IV and V arteriovenous malformations: natural history and treatment paradigm. J Neurosurg 98:3-7, 2003

8. Kano H, Flickinger JC, Yang HC, Flannery TJ, Tonetti D, Niranjan A, et al: Stereotactic radiosurgery for SpetzlerMartin Grade III arteriovenous malformations. J Neurosurg 120:973-981, 2014

9. Kano H, Lunsford LD, Flickinger JC, Yang HC, Flannery TJ, Awan NR, et al: Stereotactic radiosurgery for arteriovenous malformations, Part 1: management of Spetzler-Martin Grade I and II arteriovenous malformations. J Neurosurg 116:11-20, 2012

10. Kim H, Abla AA, Nelson J, McCulloch CE, Bervini D, Morgan MK, et al: Validation of the supplemented Spetzler-Martin grading system for brain arteriovenous malformations in a multicenter cohort of 1009 surgical patients. Neurosurgery 76:25-33, 2015

11. Korja M, Bervini D, Assaad N, Morgan MK: Role of surgery in the management of brain arteriovenous malformations: prospective cohort study. Stroke 45:3549-3555, 2014

12. Korja M, Hernesniemi J, Lawton MT, Spetzler RF, Morgan MK: Is cerebrovascular neurosurgery sacrificed on the altar of RCTs? Lancet 384:27-28, 2014 (Letter)

13. Lawton MT, Kim H, McCulloch CE, Mikhak B, Young WL: A supplementary grading scale for selecting patients with brain arteriovenous malformations for surgery. Neurosurgery 66:702-713, 2010

14. Mohr JP, Parides MK, Stapf C, Moquete E, Moy CS, Overbey JR, et al: Medical management with or without interventional therapy for unruptured brain arteriovenous malformations (ARUBA): a multicentre, non-blinded, randomised trial. Lancet 383:614-621, 2014

15. Morgan MK, Davidson AS, Koustais S, Simon M, Ritson EA: The failure of preoperative ethylene-vinyl alcohol copolymer embolization to improve outcomes in arteriovenous malformation management: case series. J Neurosurg 118:969-977, 2013

16. Morgan MK, Drummond KJ, Grinnell V, Sorby W: Surgery for cerebral arteriovenous malformation: risks related to lenticulostriate arterial supply. J Neurosurg 86:801-805, 1997

17. Morgan MK, Patel N, Simons M, Ritson EA, Heller GZ: Influence of the combination of patient age and deep venous draining on brain arteriovenous malformations recurrent after surgery. J Neurosurg 117:934-941, 2012

18. Morgan MK, Wiedmann M, Assaad NN, Heller GZ: Complication-effectiveness analysis for brain arteriovenous malformation surgery. A prospective cohort study. Neurosurgery 79:47-57, 2016

19. Morris Z, Whiteley WN, Longstreth WT Jr, Weber F, Lee YC, Tsushima Y, et al: Incidental findings on brain magnetic resonance imaging: systematic review and meta-analysis. BMJ 339:b3016, 2009 
20. Pollock BE, Flickinger JC: Modification of the radiosurgerybased arteriovenous malformation grading system. Neurosurgery 63:239-243, 2008

21. Pollock BE, Flickinger JC: A proposed radiosurgery-based grading system for arteriovenous malformations. J Neurosurg 96:79-85, 2002

22. Pollock BE, Link MJ, Stafford SL, Garces YI, Foote RL: Stereotactic radiosurgery for arteriovenous malformations: The effect of treatment period on patient outcomes. Neurosurgery 78:499-509, 2016

23. Rankin J: Cerebral vascular accidents in patients over the age of 60. II. Prognosis. Scott Med J 2:200-215, 1957

24. Spetzler RF, Martin NA: A proposed grading system for arteriovenous malformations. J Neurosurg 65:476-483, 1986

25. Spetzler RF, Ponce FA: A 3-tier classification of cerebral arteriovenous malformations. Clinical article. J Neurosurg 114:842-849, 2011

26. Stapf C, Labovitz DL, Sciacca RR, Mast H, Mohr JP, Sacco $\mathrm{RL}$ : Incidence of adult brain arteriovenous malformation hemorrhage in a prospective population-based stroke survey. Cerebrovasc Dis 13:43-46, 2002

27. Starke RM, Kano H, Ding D, Lee JYK, Mathieu D, Whitesell $\mathrm{J}$, et al: Stereotactic radiosurgery for cerebral arteriovenous malformations: evaluation of long-term outcomes in a multicenter cohort. J Neurosurg [epub ahead of print March 4, 2016. DOI: 10.3171/2015.9.JNS151311]

28. Starke RM, Yen CP, Ding D, Sheehan JP: A practical grading scale for predicting outcome after radiosurgery for arteriove- nous malformations: analysis of 1012 treated patients. J Neurosurg 119:981-987, 2013

29. van Swieten JC, Koudstaal PJ, Visser MC, Schouten HJ, van Gijn J: Interobserver agreement for the assessment of handicap in stroke patients. Stroke 19:604-607, 1988

\section{Disclosures}

The authors report no conflict of interest concerning the materials or methods used in this study or the findings specified in this paper.

\section{Author Contributions}

Conception and design: Morgan. Acquisition of data: Morgan. Analysis and interpretation of data: Morgan, Wiedmann. Drafting the article: Morgan, Wiedmann. Critically revising the article: all authors. Reviewed submitted version of manuscript: all authors. Approved the final version of the manuscript on behalf of all authors: Morgan. Statistical analysis: Morgan, Heller.

\section{Correspondence}

Michael K. Morgan, Department of Clinical Medicine, Macquarie University, 2 Technology Pl., Sydney, NSW 2109, Australia. email: michael.morgan@mq.edu.au. 